\title{
GUNDY-VAROPOULOS MARTINGALE TRANSFORMS AND THEIR PROJECTION OPERATORS ON MANIFOLDS AND VECTOR BUNDLES
}

\author{
R. BAÑUELOS, F. BAUDOIN, AND L. CHEN
}

\begin{abstract}
This paper proves the $L^{p}$ boundedness of generalized first order Riesz transforms obtained as conditional expectations of martingale transforms à la Gundy-Varopoulos for quite general diffusions on manifolds and vector bundles. Several specific examples and applications are presented: Lie groups of compact type, the Heisenberg group, SU(2), and Riesz transforms on forms and spinors.
\end{abstract}

\section{Contents}

1. Introduction

1.1. Context

1.2. Main results

2. Scalar operators constructed from martingale transforms 4

2.1. A general theorem

2.2. Example 1. Lie groups of compact type

2.3. Example 2. Heisenberg group

2.4. Example 3. $\mathbb{S U}(2)$

3. Riesz transforms on vector bundles 17

$\begin{array}{lll}\text { 3.1. A general theorem } & 17\end{array}$

3.2. Example 1. The Riesz transform on forms 19

3.3. Example 2. The Riesz transform on spinors 20

References

\section{INTRODUCTION}

1.1. Context. In Euclidean harmonic analysis, Riesz transforms play a fundamental role in singular integral theory, Hardy space theory and their applications to various areas of analysis and partial differential equations. The classical CalderónZygmund theory shows that Riesz transforms are bounded on $L^{p}$, for all $1<p<\infty$. Sharp $L^{p}$ estimates for first order Riesz transforms can be proved using either analytic [25] or probabilistic tools [7].

The study of Riesz transform in different geometric settings has a long history now with a very extensive literature. We would like to mention that Stein [41] introduced Riesz transforms on compact Lie groups and Strichartz [42] first asked

R. Bañuelos supported in part by NSF Grant 1403417-DMS. F. Baudoin supported in part by NSF Grant 1660031-DMS. 
whether one could extend their $L^{p}$ boundedness to complete Riemannian manifolds. Riesz transforms on $k$-forms associated with the Hodge Laplacian were also formulated in [42]. Ever since, there has been an explosion of literature on this topic using numerous analytic, geometric, and probabilistic methods. In the following, we will give a brief overview of previous results.

Using the martingale approach via Littlewood-Paley inequalities as in [36], Bakry [8] proved that the Riesz transforms are bounded on $L^{p}, 1<p<\infty$, on manifolds with non-negative Ricci curvature. An extension was obtained for the Riesz transform associated with the Hodge Laplacian on $L^{p}$ spaces of $k$-forms for all $1<p<\infty$ under assumption of Weitzenböck curvature. In a series of papers [30, 32, 31, 34, 33], X. D. Li gave dimension-free estimates for Riesz transforms in various geometric settings, including manifolds with assumptions on Bakry-Emery curvature or Weitzenböck curvature, and Kähler manifolds. See also [43] for the special case of the Witten Laplacians.

Another widely-adopted approach to study Riesz transform in various geometric settings is to use the Calderón-Zygmund theory for which heat kernel estimates play an essential role. See, for instance, the setting of Riemannian manifolds in $[19,27,20,29,28,3,4,18,17]$ and the references therein, especially [3, Section 1.3] with a quite complete list of previous results, and the setting of Lie groups, or manifolds with sub-elliptic operators in $[1,21,12,11]$. We note that, unlike the martingale approach, the Calderón-Zygmund theory do not give constants which are independent of dimension.

There are still many other methods to treat Riesz transforms in different settings. For instance, it was shown in [16] that the Riesz transforms on $n$-dimensional manifolds with Euclidean ends are bounded on $L^{p}$ if and only if $p \in(1, n)$. This result was generalized in $[15,23]$ and in [24] to $k$-forms for asymptotically conic manifolds. See also [37] for Riesz transforms of $k$-forms on the Heisenberg group. Last but not the least, we refer to [14] for Bellman function techniques to study Riesz transforms on manifolds under Bakry-Émery type curvature assumptions.

1.2. Main results. In the present paper, we aim to study a class of operators under very general setting which are projections of martingale transforms in the style of the now classical Gundy-Varopoulos construction on $\mathbb{R}^{d}$. We are interested in the near sharp estimates or at least dimension free estimates for these operators. More precisely, let $\mathbb{M}$ be a smooth manifold with a smooth measure $\mu$. Let $X_{1}, \cdots, X_{d}$ be locally Lipschitz vector fields defined on $\mathbb{M}$. We consider the Schrödinger operator

$$
L=-\sum_{i=1}^{d} X_{i}^{*} X_{i}+V
$$

where the $X_{i}^{*}$ denotes the formal adjoint of $X_{i}$ with respect to $\mu$ and where $V$ : $\mathbb{M} \rightarrow \mathbb{R}$ is a non-positive smooth potential. Assume that $L$ is essentially selfadjoint on the space $\mathcal{S}(\mathbb{M})$ of smooth and rapidly decreasing functions. Denote by $P_{y}=e^{-y \sqrt{-L}}$ the Poisson semigroup. For any $1 \leq i \leq d$, we consider the operator

$$
T_{i} f=\int_{0}^{+\infty} y P_{y}\left(\sqrt{-L} X_{i}-X_{i}^{*} \sqrt{-L}\right) P_{y} f d y .
$$

Our main result is the following. 
Theorem 1.1. Fix $1<p<\infty$ and set $p^{*}=\max \left\{p, \frac{p}{p-1}\right\}$. Then for every $f \in$ $\mathcal{S}(\mathbb{M})$

$$
\left\|T_{i} f\right\|_{p} \leq\left(\frac{3}{2}\right)\left(p^{*}-1\right)\|f\|_{p} .
$$

If the potential $V \equiv 0$, then

$$
\left\|T_{i} f\right\|_{p} \leq \frac{1}{2} \cot \left(\frac{\pi}{2 p^{*}}\right)\|f\|_{p} .
$$

It is interesting to note here that the constant in (1.2), up to the factor $\frac{1}{2}$, is the sharp $L^{p}$ constant for the classical Hilbert transform in $\mathbb{R}$ and the first order Riesz transforms on $\mathbb{R}^{d}$; see [38], [25], [7].

Applications of Theorem 1.1 (and of the slightly more general Theorem 2.5 given below) include, but are not limited to, the following examples.

Proposition 1.2. Let $G$ be a Lie group of compact type endowed with a bi-invariant Riemannian structure. Then

$$
\left\|X_{i}(\sqrt{-L})^{-1} f\right\|_{p} \leq \cot \left(\frac{\pi}{2 p^{*}}\right)\|f\|_{p} .
$$

The constant in the previous example is sharp and was first obtained in [2].

Proposition 1.3. Let $\mathbb{H}^{n}$ be the Heisenberg group with left invariant vector fields $X_{1}, \cdots, X_{n}, Y_{1}, \cdots, Y_{n}, Z$. Consider the sublaplacian $L=\sum_{j=1}^{n}\left(X_{j}^{2}+Y_{j}^{2}\right)$. Then

$$
\left\|\left[W_{j}, \sqrt{-L}\right] f\right\|_{p} \leq \sqrt{2}\left(p^{*}-1\right)\|Z f\|_{p},
$$

where $W_{j}=X_{j}+i Y_{j}$ is the complex gradient.

Proposition 1.4. Let $X, Y, Z$ be the left invariant vector fields on $\mathbb{S U}(2)$ and consider the sublaplacian $L=X^{2}+Y^{2}$. Then

$$
\|[W, \sqrt{-L}] f\|_{p} \leq 2 \sqrt{2}\left(p^{*}-1\right)\|(i Z+1) f\|_{p},
$$

where $W=X+i Y$ is the complex gradient.

Theorem 1.1 can also be generalized to the framework of vector bundles under general assumptions, which allows us to obtain dimension-free estimates for Riesz transforms on very general vector bundles and yields a generalization and simplification of the results in $[30,32,31,34,33]$. Let $\mathcal{E}$ be a finite-dimensional vector bundle over $\mathbb{M}$. We denote by $\Gamma(\mathbb{M}, \mathcal{E})$ the space of smooth sections of this bundle. Let now $\nabla$ denote a metric connection on $\mathcal{E}$. We consider an operator on $\Gamma(\mathbb{M}, \mathcal{E})$ that can be written as

$$
\mathcal{L}=\mathcal{F}+\nabla_{0}+\sum_{i=1}^{d} \nabla_{i}^{2},
$$

where

$$
\nabla_{i}=\nabla_{X_{i}}, \quad 0 \leq i \leq d,
$$

and the $X_{i}$ 's are smooth vector fields on $\mathbb{M}$ and $\mathcal{F}$ is a smooth symmetric and non positive potential (that is a smooth section of the bundle $\operatorname{End}(\mathcal{E})$ ). We will assume that $\mathcal{L}$ is non-positive and essentially self-adjoint on the space $\Gamma_{0}(\mathbb{M}, \mathcal{E})$ of smooth 
and compactly supported sections. We consider then a first order differential operator $d_{a}$ on $\Gamma(\mathbb{M}, \mathcal{E})$ that can be written as

$$
d_{a}=\sum_{i=1}^{d} a_{i} \nabla_{X_{i}}
$$

where $a_{1}, \cdots, a_{d}$ are smooth sections of the bundle $\operatorname{End}(\mathcal{E})$. Our main assumptions are that

$$
d_{a} \mathcal{L} \eta=\mathcal{L} d_{a} \eta, \quad \eta \in \Gamma(\mathbb{M}, \mathcal{E})
$$

and that

$$
\left\|d_{a} \eta\right\|^{2} \leq C \sum_{i=1}^{d}\left\|\nabla_{X_{i}} \eta\right\|^{2}, \quad \eta \in \Gamma(\mathbb{M}, \mathcal{E}),
$$

for some constant $C \geq 0$. Then we have the following:

Theorem 1.5. For $1<p<\infty$,

$$
\left\|d_{a}(-\mathcal{L})^{-1 / 2} \eta\right\|_{p} \leq 6 C\left(p^{*}-1\right)\|\eta\|_{p} .
$$

This result can be applied on the following examples:

(1) On Riemannian manifolds with non-negative Weitzenböck curvature. We recover then the boundedness Riesz transform on forms associated with Hodge-de Rham Laplacian as obtained by X.D. Li [30].

(2) On Riemannian manifolds which have non-negative scalar curvature and admit a spin structure. We prove then the boundedness of the Riesz transforms on spinors associated with the Dirac operators, which is a new result.

Convention: Throughout the paper, the diffusion operators we consider ( $L$ and $\mathcal{L})$ will be assumed to have no spectral gap. In the case, where those operators do have a spectral gap, all the results hold when restricting $L^{p}(\mathbb{M}, \mu)$ to the space $L_{0}^{p}(\mathbb{M}, \mu)=\left\{f \in L^{p}(\mathbb{M}, \mu), \int f d \mu=0\right\}$.

\section{SCAlAR OPERATORS CONSTRUCTED FROM MARTINGALE TRANSFORMS}

2.1. A general theorem. Let $\mathbb{M}$ be a smooth and complete Riemannian manifold with a smooth measure $\mu$. Let $X_{1}, \cdots, X_{d}$ be locally Lipschitz vector fields defined on $\mathbb{M}$. We consider the Schrödinger operator

$$
L=-\sum_{i=1}^{d} X_{i}^{*} X_{i}+V
$$

where the $X_{i}^{*}$ denotes the formal adjoint of $X_{i}$ with respect to $\mu$ and where $V$ : $\mathbb{M} \rightarrow \mathbb{R}$ is a non-positive smooth potential. Assume that $L$ is essentially self-adjoint with respect to $\mu$ on the space $\mathcal{S}(\mathbb{M})$ of smooth and rapidly decreasing functions. We denote by $\left(H_{t}\right)_{t \geq 0}$ the heat semigroup with generator $L$. We can write

$$
L=\sum_{i=1}^{d} X_{i}^{2}+X_{0}+V
$$

for some locally Lipchitz vector field $X_{0}$. 
Let $\left(Y_{t}\right)_{t \geq 0}$ be the diffusion process on $\mathbb{M}$ with generator $\sum_{i=1}^{d} X_{i}^{2}+X_{0}$ starting from the distribution $\mu$. We assume that $\left(Y_{t}\right)_{t \geq 0}$ is non explosive, it can then be constructed via the Stratonovitch stochastic differential equation

$$
d Y_{t}=X_{0}(t) d t+\sum_{i=1}^{d} X_{i}\left(Y_{t}\right) \circ d \beta_{t}^{i},
$$

where $\beta_{t}=\left(\beta_{t}^{1}, \cdots, \beta_{t}^{d}\right)$ is the Brownian motion on $\mathbb{R}^{d}$ with generator $\sum_{i=1}^{d} \frac{\partial^{2}}{\partial x_{i}^{2}}$.

Let $\left(B_{t}\right)_{t \geq 0}$ be a one-dimensional Brownian motion on $\mathbb{R}$, with generator $\frac{d^{2}}{d y^{2}}$ starting from $y_{0}>0$, which is independent from $\left(Y_{t}\right)_{t \geq 0}$. Then $\mathbb{E}\left(B_{t}^{2}\right)=2 t$. Set the stopping time

$$
\tau=\inf \left\{t>0, B_{t}=0\right\} .
$$

We denote by $H_{t}=e^{t L}$ the heat semigroup and by $P_{y}=e^{-y \sqrt{-L}}$ the Poisson semigroup. By the Feynman-Kac formula, the heat semigroup $H_{t}$ acting on $f \in$ $\mathcal{S}(\mathbb{M})$ can be written as

$$
H_{t} f(x)=\mathbb{E}_{x}\left(e^{\int_{0}^{t} V\left(Y_{s}\right) d s} f\left(Y_{t}\right)\right) .
$$

To work on a set of test functions which is large enough, we assume that $\mathcal{S}(\mathbb{M})$ is stable by $H_{t}, P_{y}, X_{i}, X_{i}^{*}$ and $\sqrt{-L}$. Let $1 \leq i \leq d$. Consider the operator

$$
T_{i}=\int_{0}^{+\infty} y P_{y}\left(\sqrt{-L} X_{i}-X_{i}^{*} \sqrt{-L}\right) P_{y} d y .
$$

We assume that $T_{i}$ is pointwisely well defined on $\mathcal{S}(\mathbb{M})$ and $T_{i}(\mathcal{S}(\mathbb{M})) \subset \mathcal{S}(\mathbb{M})$. We have then the following Gundy-Varopoulos type probabilistic representation of $T_{i}$.

Lemma 2.1. Let $f \in \mathcal{S}(\mathbb{M})$. For almost all $x \in \mathbb{M}$, we have, for $i=1,2, \ldots, d$,

$T_{i} f(x)=-\frac{1}{2} \lim _{y_{0} \rightarrow \infty} \mathbb{E}_{y_{0}}\left(e^{\int_{0}^{\tau} V\left(Y_{v}\right) d v} \int_{0}^{\tau} e^{-\int_{0}^{s} V\left(Y_{v}\right) d v} A_{i}\left(\nabla, \partial_{y}\right)^{\mathrm{T}} Q^{V} f\left(Y_{s}, B_{s}\right)\left(d \beta_{s}, d B_{s}\right) \mid Y_{\tau}=x\right)$,

where $\nabla=\left(X_{1}, \cdots, X_{d}\right)$, and $A_{i}$ is a $(d+1) \times(d+1)$ matrix with $a_{i(d+1)}=-1$, $a_{(d+1) i}=1$ and otherwise 0 .

To prove the lemma, we introduce some notation. For $f \in \mathcal{S}(\mathbb{M})$, we denote

$$
Q^{V} f(x, y)=P_{y} f(x)=e^{-y \sqrt{-L}} f(x),
$$

and

$$
M_{t}^{f}=e^{\int_{0}^{t \wedge \tau} V\left(Y_{s}\right) d s} Q^{V} f\left(Y_{t \wedge \tau}, B_{t \wedge \tau}\right) .
$$

Lemma 2.2. Let $f \in \mathcal{S}(\mathbb{M})$. The process $M_{t}^{f}$ is a square integrable martingale. Moreover, the quadratic variation is

$$
\begin{aligned}
\left\langle M^{f}\right\rangle_{t}= & 2 \int_{0}^{t \wedge \tau}\left(e^{\int_{0}^{\tau} V\left(Y_{v}\right) d v} \partial_{y} Q^{V} f\left(Y_{s}, B_{s}\right)\right)^{2} d s \\
& +2 \int_{0}^{t \wedge \tau} \sum_{i=1}^{d}\left(e^{\int_{0}^{\tau} V\left(Y_{v}\right) d v} X_{i} Q^{V} f\left(Y_{s}, B_{s}\right)\right)^{2} d s
\end{aligned}
$$

Proof. We follow the proof in [35]. Note that

$$
M_{\tau}^{f}=e^{\int_{0}^{\tau} V\left(Y_{v}\right) d v} Q^{V} f\left(Y_{\tau}, 0\right)=e^{\int_{0}^{\tau} V\left(Y_{u}\right) d u} f\left(Y_{\tau}\right) .
$$


Let $\lambda_{u}$ be the distribution of $\tau$ at $B_{0}=u$. Since the processes $Y_{t}$ and $B_{t}$ are independent, then by the Feynman-Kac formula,

$$
\begin{aligned}
\mathbb{E}\left(e^{\int_{0}^{\tau} V\left(Y_{v}\right) d v} f\left(Y_{\tau}\right) \mid Y_{0}=x, B_{0}=u\right) & =\int_{0}^{\infty} \mathbb{E}\left(e^{\int_{0}^{s} V\left(Y_{v}\right) d v} f\left(Y_{s}\right) \mid Y_{0}=x\right) \lambda_{u}(d s) \\
& =\int_{0}^{\infty} H_{s} f(y) \lambda_{u}(d s)=Q^{V} f(x, u) .
\end{aligned}
$$

The last equality above follows from the subordination formula due to the fact that

$$
\lambda_{u}(d s)=\frac{u}{2 \sqrt{\pi}} e^{-u^{2} / 4 s} s^{-3 / 2} d s .
$$

Therefore we have

$$
\begin{aligned}
\mathbb{E}\left(M_{\tau}^{f} \mid \mathcal{F}_{s}\right) & =\mathbb{E}\left(e^{\int_{0}^{\tau} V\left(Y_{v}\right) d v} f\left(Y_{\tau}\right) \mid \mathcal{F}_{s}\right) \\
& =\mathbb{E}\left(e^{\int_{0}^{\tau} V\left(Y_{v}\right) d v} f\left(Y_{\tau}\right) 1_{\tau \leq s} \mid \mathcal{F}_{s}\right)+\mathbb{E}\left(e^{\int_{0}^{\tau} V\left(Y_{v}\right) d v} f\left(Y_{\tau}\right) 1_{\tau>s} \mid \mathcal{F}_{s}\right) \\
& =e^{\int_{0}^{\tau} V\left(Y_{v}\right) d v} f\left(Y_{\tau}\right) 1_{\tau \leq s}+\mathbb{E}\left(e^{\int_{0}^{\tau} V\left(Y_{v}\right) d v} f\left(Y_{\tau}\right) 1_{\tau>s} \mid\left(Y_{s}, B_{s}\right)\right)=M_{s}^{f} .
\end{aligned}
$$

Now applying Itô's formula, we obtain that for any $0<t \leq \tau$,

$$
\begin{aligned}
e^{\int_{0}^{t} V\left(Y_{v}\right) d v} Q^{V} f\left(Y_{t}, B_{t}\right)= & Q^{V} f\left(Y_{0}, B_{0}\right)+\sum_{i=1}^{d} \int_{0}^{\tau} e^{\int_{0}^{s} V\left(Y_{v}\right) d v} X_{i} Q^{V} f\left(Y_{s}, B_{s}\right) d \beta_{s}^{i} \\
& +\int_{0}^{\tau} e^{\int_{0}^{s} V\left(Y_{v}\right) d v} \partial_{y} Q^{V} f\left(Y_{s}, B_{s}\right) d B_{s} .
\end{aligned}
$$

Hence

$$
\begin{aligned}
\left\langle M^{f}\right\rangle_{t}= & 2 \int_{0}^{t \wedge \tau}\left(e^{\int_{0}^{\tau} V\left(Y_{v}\right) d v} \partial_{y} Q^{V} f\left(Y_{s}, B_{s}\right)\right)^{2} d s \\
& +2 \int_{0}^{t \wedge \tau} \sum_{i=1}^{d}\left(e^{\int_{0}^{\tau} V\left(Y_{v}\right) d v} X_{i} Q^{V} f\left(Y_{s}, B_{s}\right)\right)^{2} d s
\end{aligned}
$$

Proof of Lemma 2.1. Let $f \in \mathcal{S}(\mathbb{M})$ and $g \in \mathcal{S}(\mathbb{M})$. Note that $Y$ and $B$ are independent and recall that

$$
M_{\tau}^{g}=e^{\int_{0}^{\tau} V\left(Y_{v}\right) d v} g\left(Y_{\tau}\right) .
$$

By Itô's formula (2.3) and the Itô isometry, we have

$$
\begin{aligned}
& \int_{\mathbb{M}} g(x)\left(e^{\int_{0}^{\tau} V\left(Y_{v}\right) d v} \int_{0}^{\tau} e^{-\int_{0}^{s} V\left(Y_{v}\right) d v} A_{i}\left(\nabla, \partial_{y}\right)^{\mathrm{T}} Q^{V} f\left(Y_{s}, B_{s}\right)\left(d \beta_{s}, d B_{s}\right) \mid Y_{\tau}=x\right) d \mu(x) \\
& =\mathbb{E}_{y_{0}}\left(g\left(Y_{\tau}\right) e^{\int_{0}^{\tau} V\left(Y_{v}\right) d v} \int_{0}^{\tau} e^{-\int_{0}^{s} V\left(Y_{v}\right) d v} A_{i}\left(\nabla, \partial_{y}\right)^{\mathrm{T}} Q^{V} f\left(Y_{s}, B_{s}\right)\left(d \beta_{s}, d B_{s}\right)\right) \\
& =2 \mathbb{E}_{y_{0}}\left(\int_{0}^{\tau} \partial_{y} Q^{V} g\left(Y_{s}, B_{s}\right) X_{i} Q^{V} f\left(Y_{s}, B_{s}\right) d s-\int_{0}^{\tau} X_{i} Q^{V} g\left(Y_{s}, B_{s}\right) \partial_{y} Q^{V} f\left(Y_{s}, B_{s}\right) d s\right) \\
& =2 \int_{\mathbb{M}} \int_{0}^{\infty}\left(y_{0} \wedge y\right)\left(\partial_{y} Q^{V} g(x, y) X_{i} Q^{V} f(x, y)-X_{i} Q^{V} g(x, y) \partial_{y} Q^{V} f(x, y)\right) d y d \mu(x) .
\end{aligned}
$$

The last equality follows from the facts that the Green function of the Brownian motion is killed at 0 and $Y_{s}$ is distributed according to $\mu$. 
Since $L$ is self-adjoint, then

$\int_{\mathbb{M}} \int_{0}^{\infty} y \partial_{y} Q^{V} g(x, y) X_{i} Q^{V} f(x, y) d y d \mu(x)=-\int_{\mathbb{M}} g(x) \int_{0}^{\infty} y P_{y} \sqrt{-L} X_{i} P_{y} f(x) d y d \mu(x)$,

and

$\int_{\mathbb{M}} \int_{0}^{\infty} y X_{i} Q^{V} g(x, y) \partial_{y} Q^{V} f(x, y) d y d \mu(x)=-\int_{\mathbb{M}} g(x) \int_{0}^{\infty} y P_{y} X_{i}^{*} \sqrt{-L} P_{y} f(x) d y d \mu(x)$.

Therefore we obtain

$T_{i} f(x)=-\frac{1}{2} \lim _{y_{0} \rightarrow \infty} \mathbb{E}_{y_{0}}\left(e^{\int_{0}^{\tau} V\left(Y_{v}\right) d v} \int_{0}^{\tau} e^{-\int_{0}^{s} V\left(Y_{v}\right) d v} A_{i}\left(\nabla, \partial_{y}\right)^{\mathrm{T}} Q^{V} f\left(Y_{s}, B_{s}\right)\left(d \beta_{s}, d B_{s}\right) \mid Y_{\tau}=x\right)$.

To study the $L^{p}$ boundedness of $T_{i}$, we shall use some extensions of Burkholder's celebrated theorem on martingale transforms. Before stating the results, we introduce some necessary probabilistic background. Suppose that $(\Omega, \mathcal{F}, \mathbb{P})$ is a complete probability space, filtered by $\mathcal{F}=\left\{\mathcal{F}_{t}\right\}_{t \geq 0}$, a family of right continuous sub- $\sigma$-fields of $\mathcal{F}$. Assume that $\mathcal{F}_{0}$ contains all the events of probability 0 . Let $X$ and $Y$ be adapted, real-valued martingales which have right-continuous paths with left-limits (r.c.l.l.). The martingale $Y$ is differentially subordinate to $X$ if $\left|Y_{0}\right| \leq\left|X_{0}\right|$ and $\langle X\rangle_{t}-\langle Y\rangle_{t}$ is a nondecreasing and nonnegative function of $t$. The martingales $X_{t}$ and $Y_{t}$ are said to be orthogonal if the covariation process $\langle X, Y\rangle_{t}=0$ for all $t$. Bañuelos and Wang [7] proved the following sharp inequality extending the classical results of Burkholder. We always assume the martingale $X$ (hence $Y$ ) is $L^{p}$ bounded for $1<p<\infty$ and by $X$ in he inequalities below we mean $X_{\infty}$. Similarly for $Y$.

Theorem 2.3. Let $X$ and $Y$ be two martingales with continuous paths such that $Y$ is differentially subordinate to $X$. Fix $1<p<\infty$ and set $p^{*}=\max \left\{p, \frac{p}{p-1}\right\}$. Then

$$
\|Y\|_{p} \leq\left(p^{*}-1\right)\|X\|_{p} .
$$

Furthermore, suppose the martingales $X$ and $Y$ are orthogonal. Then

$$
\|Y\|_{p} \leq \cot \left(\frac{\pi}{2 p^{*}}\right)\|X\|_{p}
$$

Both of these inequalities are sharp.

More generally, Bañuelos and Osȩkowski [6] proved that

Theorem 2.4. Let $X$ and $Y$ be two martingales with continuous paths such that $Y$ is differentially subordinate to $X$. Consider the process

$$
Z_{t}=e^{\int_{0}^{t} V_{s} d s} \int_{0}^{t} e^{-\int_{0}^{s} V_{v} d v} d Y_{s}
$$

where $\left(V_{t}\right)_{t \geq 0}$ is a non-positive adapted and continuous process. For $1<p<\infty$, we have the sharp bound

$$
\|Z\|_{p} \leq\left(p^{*}-1\right)\|X\|_{p}
$$


Proof of Theorem 1.1. We first consider the case that $V \equiv 0$. Note that the martingale

$$
N_{t}=\int_{0}^{t \wedge \tau} A_{i}\left(\nabla, \partial_{y}\right)^{\mathrm{T}} Q f\left(Y_{s}, B_{s}\right)\left(d \beta_{s}, d B_{s}\right)
$$

is differentially subordinate to $M_{t}^{f}=Q f\left(Y_{t \wedge \tau}, B_{t \wedge \tau}\right)$. In addition, since the matrix $A_{i}$ is orthogonal, that is, $\langle A v, v\rangle=0$ for all $v \in \mathbb{R}^{d+1},\left\langle M^{f}, N\right\rangle_{t}=0$. Hence Lemma 2.1 and Theorem 2.3 gives us

$$
\left\|T_{i} f\right\|_{p} \leq \frac{1}{2} \cot \left(\frac{\pi}{2 p^{*}}\right)\|f\|_{p} .
$$

Next we deal with the case $V \neq 0$. The stochastic integral

$$
\int_{0}^{t \wedge \tau} A_{i}\left(\nabla, \partial_{y}\right)^{\mathrm{T}} Q^{V} f\left(Y_{s}, B_{s}\right)\left(d \beta_{s}, d B_{s}\right)
$$

is subordinate to

$$
\int_{0}^{t \wedge \tau}\left(\nabla, \partial_{y}\right)^{\mathrm{T}} Q^{V} f\left(Y_{s}, B_{s}\right)\left(d \beta_{s}, d B_{s}\right) .
$$

Using Itô's formula for $Q^{V} f\left(Y_{t}, B_{t}\right)$, we have

$$
\begin{aligned}
Q^{V} f\left(Y_{t \wedge \tau}, B_{t \wedge \tau}\right)= & Q^{V} f\left(Y_{0}, B_{0}\right)+\int_{0}^{t \wedge \tau}\left(\nabla, \partial_{y}\right)^{\mathrm{T}} Q^{V} f\left(Y_{s}, B_{s}\right)\left(d \beta_{s}, d B_{s}\right) \\
& +2 \int_{0}^{t \wedge \tau}\left(\partial_{y}^{2}+\sum_{i=1}^{d} X_{i}^{2}+X_{0}\right) Q^{V} f\left(Y_{s}, B_{s}\right) d s .
\end{aligned}
$$

Since $Q^{V} f(x, y)=e^{-y \sqrt{-L}} f(x)$ satisfies

$$
\left(\partial_{y}^{2}+\sum_{i=1}^{d} X_{i}^{2}+X_{0}\right) Q^{V} f=-V Q^{V} f
$$

then we get

$$
\begin{aligned}
Q^{V} f\left(Y_{t \wedge \tau}, B_{t \wedge \tau}\right)=Q^{V} f\left(Y_{0}, B_{0}\right) & +\int_{0}^{t \wedge \tau}\left(\nabla, \partial_{y}\right)^{\mathrm{T}} Q^{V} f\left(Y_{s}, B_{s}\right)\left(d \beta_{s}, d B_{s}\right) \\
& -2 \int_{0}^{t \wedge \tau} V\left(Y_{s}\right) Q^{V} f\left(Y_{s}, B_{s}\right) d s .
\end{aligned}
$$

Suppose $f \geq 0$. Then $Q^{V} f\left(Y_{t \wedge \tau}, B_{t \wedge \tau}\right)$ is a non-negative submartingale. It follows from Lenglart-Lépingle-Pratelli [26, Theorem 3.2, part 3)] that

$$
\begin{aligned}
\left\|Q^{V} f\left(Y_{0}, B_{0}\right)-2 \int_{0}^{\tau} V\left(Y_{s}\right) Q^{V} f\left(Y_{s}, B_{s}\right) d s\right\|_{p} & \leq p\left\|Q^{V} f\left(Y_{\tau}, B_{\tau}\right)\right\| \\
& =p\left\|f\left(Y_{\tau}\right)\right\|_{p}=p\|f\|_{p} .
\end{aligned}
$$

This yields

$$
\left\|\int_{0}^{\tau}\left(\nabla, \partial_{y}\right)^{\mathrm{T}} Q^{V} f\left(Y_{s}, B_{s}\right)\left(d \beta_{s}, d B_{s}\right)\right\| \leq(p+1)\|f\|_{p} .
$$

For a general $f$, write

$$
A_{t} f:=Q^{V} f\left(Y_{0}, B_{0}\right)-2 \int_{0}^{t \wedge \tau} V\left(Y_{s}\right) Q^{V} f\left(Y_{s}, B_{s}\right) d s .
$$


Notice that since $\left|Q^{V} f\right| \leq Q^{V}|f|$ and $V$ is non-positive, then we have $\left|A_{t} f\right| \leq A_{t}|f|$ and the above argument shows that (2.7) holds for general $f$.

We now assume that $1<p \leq 2$. Applying Lemma 2.1 and Theorem 2.4, we conclude that

$$
\left\|T_{i} f\right\|_{p} \leq 3\left(\frac{p^{*}-1}{2}\right)\|f\|_{p}, \quad 1<p \leq 2 .
$$

To deal with the case of $2 \leq p<\infty$, we recall (as in [5]), that if $T_{A}$ is the operator constructed as above with $A_{i}$ replaced by a general $(d+1) \times(d+1)$ matrix $A$, its adjoint, $T_{A}^{*}$ is given by $T_{A} *$. Thus, by duality, using the fact that $\left(p^{*}-1\right)$ equals $(p-1)$, for $2 \leq p<\infty$, and equals $\frac{1}{(p-1)}$, for $1<p \leq 2$, we get

$$
\left\|T_{i} f\right\|_{p} \leq 3\left(\frac{p^{*}-1}{2}\right)\|f\|_{p}, \quad 2<p \leq \infty .
$$

The estimates (2.8) and (2.9) give the estimate (1.1).

The same proof above gives the following more general result for conditional expectations (projections) of martingale transforms as above.

Theorem 2.5. Let $f \in \mathcal{S}(\mathbb{M})$ and let $A$ be $a(d+1) \times(d+1)$ matrix of norm $\|A\|$. Then for $1<p<\infty$,

$T_{A} f(x)=-\frac{1}{2} \lim _{y_{0} \rightarrow \infty} \mathbb{E}_{y_{0}}\left(e^{\int_{0}^{\tau} V\left(Y_{v}\right) d v} \int_{0}^{\tau} e^{-\int_{0}^{s} V\left(Y_{v}\right) d v} A\left(\nabla, \partial_{y}\right)^{\mathrm{T}} Q^{V} f\left(Y_{s}, B_{s}\right)\left(d \beta_{s}, d B_{s}\right) \mid Y_{\tau}=x\right)$, satisfies

$$
\left\|T_{A} f\right\|_{p} \leq\|A\|\left(\frac{3}{2}\right)\left(p^{*}-1\right)\|f\|_{p}
$$

and if $V \equiv 0$,

$$
\left\|T_{A} f\right\|_{p} \leq\|A\| \frac{\left(p^{*}-1\right)}{2}\|f\|_{p} .
$$

2.2. Example 1. Lie groups of compact type. Let $G$ be a Lie group of compact type with Lie algebra $\mathfrak{g}$. We endow $G$ with a bi-invariant Riemannian structure and consider an orthonormal basis $X_{1}, \cdots, X_{d}$ of $\mathfrak{g}$. In this setting the Laplace-Beltrami operator can be written as

$$
L=\sum_{i=1}^{d} X_{i}^{2}
$$

It is essentially self-adjoint on the space of smooth and compactly supported functions. Then $X_{i}^{*}=-X_{i}$ and $X_{i}$ commutes with $P_{y}$. We easily see that

$T_{i}=\int_{0}^{+\infty} y P_{y}\left(\sqrt{-L} X_{i}-X_{i}^{*} \sqrt{-L}\right) P_{y} d y=2 X_{i} \sqrt{-L} \int_{0}^{+\infty} y P_{y} P_{y} d y=\frac{1}{2} X_{i}(\sqrt{-L})^{-1}$.

As a consequence of Lemma 2.1 and Theorem 1.1, the Riesz transform is bounded on $L^{p}(M)$ and we have the estimate

$$
\left\|X_{i}(\sqrt{-L})^{-1}\right\|_{L^{p} \rightarrow L^{p}} \leq \cot \left(\frac{\pi}{2 p^{*}}\right) .
$$

This inequality was first proved in [2] where the proof is also based on the martingale inequality (2.5) 
2.3. Example 2. Heisenberg group. Another interesting example is given by the Heisenberg group. The Heisenberg group is the set

$$
\mathbb{H}^{n}=\left\{(x, y, z): x \in \mathbb{R}^{n}, y \in \mathbb{R}^{n}, z \in \mathbb{R}\right\}
$$

endowed with the group law

$$
(x, y, z) \cdot\left(x^{\prime}, y^{\prime}, z^{\prime}\right)=\left(x+x^{\prime}, y+y^{\prime}, z+z^{\prime}+\frac{1}{2}\left(\left\langle x, y^{\prime}\right\rangle_{\mathbb{R}^{n}}-\left\langle y, x^{\prime}\right\rangle_{\mathbb{R}^{n}}\right)\right) .
$$

Consider the left-invariant vector fields: for any $j \in \mathbb{N}, 1 \leq j \leq n$,

$$
X_{j}=\partial_{x_{j}}-\frac{y_{j}}{2} \partial_{z}, \quad Y_{j}=\partial_{y_{j}}+\frac{x_{j}}{2} \partial_{z}, \quad Z=\partial_{z},
$$

and the sublaplacian

$$
L=\sum_{j=1}^{n}\left(X_{j}^{2}+Y_{j}^{2}\right) .
$$

Denote by $\mathcal{S}\left(\mathbb{H}^{n}\right)\left(=\mathcal{S}\left(\mathbb{R}^{2 n+1}\right)\right)$ the Schwartz space of smooth rapidly decreasing functions on the Heisenberg group. Equivalently,

$$
\mathcal{S}\left(\mathbb{H}^{n}\right)=\left\{f \in C^{\infty}\left(\mathbb{H}^{n}\right): \sup _{\mathbf{x} \in \mathbb{H}}(1+|\mathbf{x}|)^{q}\left|X^{K} f(\mathbf{x})\right|<\infty, \forall K \in\left(\mathbb{N}^{*}\right)^{2 n}, \forall q \in \mathbb{N}\right\}
$$

where $|\mathbf{x}|=\left(\left(x^{2}+y^{2}\right)^{2}+z^{2}\right)^{1 / 4}, \mathbb{N}^{*}=\mathbb{N} \cup\{0\}$ and

$$
X^{K}=X_{1}^{k_{1}} \cdots X_{n}^{k_{n}} Y_{1}^{k_{n+1}} \cdots Y_{n}^{k_{2 n}}, \quad \text { where } K=\left(k_{1}, k_{2}, \cdots, k_{2 n}\right) \in\left(\mathbb{N}^{*}\right)^{2 n} .
$$

Let $d(\mathbf{x}, \mathbf{y})$ be the Carnot-Carathéodory distance. Notice that $|\mathbf{x}| \simeq d(0, \mathbf{x})$.

The sublaplacian $L$ is essentially self-adjoint on $\mathcal{S}\left(\mathbb{H}^{n}\right)$. Denote by $[U, V]=$ $U V-V U$ the commutator of $U$ and $V$, then for any $1 \leq j, k \leq n$,

$$
\left[X_{j}, Y_{k}\right]=\delta_{j k} Z, \quad\left[X_{j}, Z\right]=0, \quad\left[Y_{k}, Z\right]=0 .
$$

Let $W_{j}=X_{j}+i Y_{j}$ be the complex gradient, then

$$
W_{j} L=(L-2 i Z) W_{j} .
$$

In other words, we have $\left[W_{j}, L\right]=-2 i Z W_{j}$. Note also that $\left[W_{j}, Z\right]=0$ and $[L, Z]=0$.

The right invariant vector fields are given by

$$
\hat{X}_{j}=\partial_{x_{j}}+\frac{y_{j}}{2} \partial_{z}, \quad \hat{Y}_{j}=\partial_{y_{j}}-\frac{x_{j}}{2} \partial_{z}, \quad \hat{Z}=\partial_{z} .
$$

Then we have the right-invariant sublaplacian and complex gradient, denoted by $\hat{L}$ and $\hat{W}_{j}$, respectively.

Let $\left(H_{t}\right)_{t>0}=\left(e^{t L}\right)_{t>0}$ be the heat semigroup generated by $L$ and $h_{t}(\mathbf{x})$ be the corresponding heat kernel at 0 . Hence $h_{t}(\mathbf{x}, \mathbf{y})=h_{t}\left(\mathbf{x y}^{-1}\right)$. Let $\left(P_{t}\right)_{t>0}=$ $\left(e^{-t \sqrt{-L}}\right)_{t>0}$ be the Poisson semigroup and $p_{t}$ be the Poisson kernel at 0. Similarly, let $\left(\hat{H}_{t}\right)_{t>0}=\left(e^{t \hat{L}}\right)_{t>0}$ and $\left(\hat{P}_{t}\right)_{t>0}=\left(e^{-t \sqrt{-\hat{L}}}\right)_{t>0}$ be the heat and Poisson semigroups generated by $\hat{L}$. The corresponding heat and Poisson kernels are denoted by $\hat{h}_{t}$ and $\hat{p}_{t}$.

We have that $\mathcal{S}\left(\mathbb{H}^{n}\right)$ is left globally stable by $L$ and by $H_{t}$ for any $t \geq 0$ (see $[9$, Lemma 2.1]). Moreover, define $\sqrt{-L} f$ via the heat semigroup as follows:

$$
\sqrt{-L} f(\mathbf{x})=-\frac{1}{2 \sqrt{\pi}} \int_{0}^{\infty} t^{-3 / 2}\left(H_{t} f(\mathbf{x})-f(\mathbf{x})\right) d t .
$$

Then 
Lemma 2.6. For any $f \in \mathcal{S}\left(\mathbb{H}^{n}\right)$, we have $\sqrt{-L} f \in \mathcal{S}\left(\mathbb{H}^{n}\right)$.

Proof. Let $f \in \mathcal{S}\left(\mathbb{H}^{n}\right)$, then for any $K=\left(k_{1}, k_{2}, \cdots, k_{2 n}\right) \in\left(\mathbb{N}^{*}\right)^{2 n}$ and $q \in \mathbb{N}$,

$$
\left|\hat{X}^{K} f(\mathbf{x})\right| \leq \frac{C}{\left(1+d^{2}(0, \mathbf{x})\right)^{q}},
$$

where $\hat{X}^{K}=\hat{X}_{1}^{k_{1}} \cdots \hat{X}_{n}^{k_{n}} \hat{Y}_{1}^{k_{n+1}} \cdots \hat{Y}_{n}^{k_{2 n}}$.

Following the argument in [9], we have that for any $t \geq 1$

$$
\begin{aligned}
\left|X^{K} H_{t} f(0)\right| & =\left|H_{t} \hat{X}^{K} f(0)\right| \leq H_{t}\left(\frac{C}{\left(1+d^{2}(0, \cdot)\right)^{q}}\right)(0) \leq \mathbb{E}_{0}\left(\frac{C}{\left(1+d^{2}\left(0, X_{t}\right)\right)^{q}}\right) \\
& \leq \mathbb{E}_{0}\left(\frac{C}{\left(1+t d^{2}\left(0, X_{1}\right)\right)^{q}}\right) \leq \frac{C}{t^{q}} \mathbb{E}_{0}\left(\frac{1}{\left(1+d^{2}\left(0, X_{1}\right)\right)^{q}}\right) \\
& \leq \frac{C}{t^{q}} H_{1}\left(\frac{C}{\left(1+d^{2}(0, \cdot)\right)^{q}}\right)(0) .
\end{aligned}
$$

By the left invariance, we obtain that

$$
\left|X^{K} H_{t} f(x)\right|=\left|H_{t} \hat{X}^{K} f(0)\right| \leq \frac{C}{t^{q}}\left(1+d^{2}(0, \mathbf{x})\right)^{-q}
$$

Also recall that for $0<t<1$,

$$
\left|X^{K} H_{t} f\right|=\left|H_{t} \hat{X}^{K} f\right| \leq e^{C t}\left(1+d^{2}(0, \mathbf{x})\right)^{-q} .
$$

Ignoring the constant, we rewrite (2.13) as

$$
\sqrt{-L} f(\mathbf{x})=\int_{0}^{1} t^{-3 / 2} \int_{0}^{t} L H_{s} f(\mathbf{x}) d s d t+\int_{1}^{\infty} t^{-3 / 2}\left(H_{t} f(\mathbf{x})-f(\mathbf{x})\right) d t .
$$

Using (2.15) and (2.14), we obtain

$$
\sup _{\mathbf{x} \in \mathbb{H}}(1+|\mathbf{x}|)^{q}\left|X^{K} \sqrt{-L} f(\mathbf{x})\right| \lesssim \int_{0}^{1} t^{-1 / 2} d t+\int_{1}^{\infty} t^{-3 / 2} d t<\infty .
$$

Remark 2.7. Consequently, $\left[W_{j}, \sqrt{-L}\right]$ is defined pointwisely on $\mathcal{S}\left(\mathbb{H}^{n}\right)$. Similarly, we can also show that $2 i T_{j} Z f \in \mathcal{S}\left(\mathbb{H}^{n}\right)$ by using the subordination formula for the Poisson semigroup

$$
P_{t} f=\frac{t}{2 \sqrt{\pi}} \int_{0}^{\infty} e^{-\frac{t^{2}}{4 s}} H_{s} f \frac{d s}{s^{3 / 2}} .
$$

Consider the operator

$$
\mathcal{T}_{j}=\int_{0}^{+\infty} y P_{y}\left(W_{j} \sqrt{-L}+\sqrt{-L} W_{j}\right) P_{y} d y
$$

we have the following equality.

Proposition 2.8. For any $f \in \mathcal{S}\left(\mathbb{H}^{n}\right)$, there holds

$$
\left[W_{j}, \sqrt{-L}\right] f=2 i \mathcal{T}_{j} Z f .
$$

In order to prove Proposition 2.8, we recall first the spectral decomposition of the sublaplacian on the Heisenberg group (see, for instance, [39, Section 2]). Let $f \in L^{2}\left(\mathbb{H}^{n}\right)$ be a radial function. That is, for any $\mathbf{x}=(x, y, z) \in \mathbb{H}^{n}, f(\mathbf{x})=f(r, z)$ 
with $r=\|\mathbf{x}\|$. Here $\|\mathbf{x}\|$ denotes the Euclidean norm of the projection of $\mathbf{x}$ onto the plane $\{z=0\}$. The spectral decomposition of the sublaplacian is given by

$$
L f(r, z)=-(2 \pi)^{-n-1} \int_{-\infty}^{\infty} \sum_{k=0}^{\infty}(2 k+n)|\lambda| C_{k}^{\lambda}\left(f^{\lambda}\right) \varphi_{k}^{\lambda}(r) e^{-i \lambda z}|\lambda|^{n} d \lambda,
$$

where $\varphi_{k}^{\lambda}$ are the scaled Laguerre functions

$$
\varphi_{k}^{\lambda}(r)=L_{k}^{n-1}\left(\frac{1}{2}|\lambda| r^{2}\right) e^{-\frac{1}{4}|\lambda| r^{2}},
$$

and $C_{k}^{\lambda}\left(f^{\lambda}\right)$ are the Laguerre coefficients of the radial function $f^{\lambda}$ given by

$$
C_{k}^{\lambda}\left(f^{\lambda}\right)=C_{n, \lambda} \frac{k !(n-1) !}{(k+n-1) !} \int_{\mathbb{R}^{2}} f^{\lambda}(r) \varphi_{k}^{\lambda}(r) d x d y .
$$

Notice that $\left\{\varphi_{k}^{\lambda}\right\}_{k=0}^{\infty}$ forms an orthogonal basis for the subspace consisting of radial functions in $L^{2}\left(\mathbb{R}^{2 n}\right)$. The spectral decomposition of the associated heat semigroup is

$$
e^{t L} f(r, z)=(2 \pi)^{-n-1} \int_{-\infty}^{\infty} \sum_{k=0}^{\infty} e^{-(2 k+n)|\lambda| t} C_{k}^{\lambda}\left(f^{\lambda}\right) \varphi_{k}^{\lambda}(r) e^{-i \lambda z}|\lambda|^{n} d \lambda .
$$

We also have the spectral decomposition for the heat kernel

$$
h_{t}(r, z)=C \int_{-\infty}^{\infty} \sum_{k=0}^{\infty} e^{-(2 k+n)|\lambda| t} \varphi_{k}^{\lambda}(r) e^{-i \lambda z}|\lambda|^{n} d \lambda .
$$

Lemma 2.9. For any $r>0, z \in \mathbb{R}$ and $t>0$, we have

$$
\left[W_{j}, \sqrt{-L}\right] h_{t}(r, z)=\int_{-\infty}^{\infty} \sum_{k=0}^{\infty} e^{-(2 k+n)|\lambda| t}\left[W_{j}, \sqrt{-L}\right] \varphi_{k}^{\lambda}(r) e^{-i \lambda z}|\lambda|^{n} d \lambda,
$$

where the right hand side converges uniformly in $r$ and $z$.

Proof. For any $\mathbf{x}=(x, y, z) \in \mathbb{H}$, denote $\widetilde{\varphi}_{k}^{\lambda}(\mathbf{x})=\widetilde{\varphi}_{k}^{\lambda}(r, z)=\varphi_{k}^{\lambda}(r) e^{-i \lambda z}|\lambda|$, where $r=\|\mathbf{x}\|$. Then for any $t_{0}>0$

$$
H_{t_{0}} \widetilde{\varphi}_{k}^{\lambda}=e^{-(2 k+n)|\lambda| t_{0}} \widetilde{\varphi}_{k}^{\lambda}
$$

and

$$
W_{j} \widetilde{\varphi}_{k}^{\lambda}(\mathbf{x})=e^{(2 k+n)|\lambda| t_{0}} W_{j} H_{t_{0}} \widetilde{\varphi}_{k}^{\lambda}(\mathbf{x})=e^{(2 k+n)|\lambda| t_{0}} \int_{\mathbb{H}^{n}} W_{j ; \mathbf{x}} h_{t_{0}}(\mathbf{x}, \mathbf{y}) \widetilde{\varphi}_{k}^{\lambda}(\mathbf{y}) d \mathbf{y} .
$$

Consequently,

$$
\begin{aligned}
\left|W_{j} \sqrt{-L} \widetilde{\varphi}_{k}^{\lambda}(\mathbf{x})\right| & =\sqrt{(2 k+n)|\lambda|} e^{(2 k+n)|\lambda| t_{0}}\left|\int_{\mathbb{H}^{n}} W_{j ; \mathbf{x}} h_{t_{0}}(\mathbf{x}, \mathbf{y}) \widetilde{\varphi}_{k}^{\lambda}(\mathbf{y}) d \mathbf{y}\right| \\
& \leq \sqrt{(2 k+n)|\lambda|} e^{(2 k+n)|\lambda| t_{0}}\left\|W_{j ; \mathbf{x}} h_{t_{0}}(\mathbf{x}, \cdot)\right\|_{L^{1}}\left\|\widetilde{\varphi}_{k}^{\lambda}\right\|_{L^{\infty}} \\
& \leq C t_{0}^{-1 / 2} \sqrt{(2 k+n)|\lambda|} e^{(2 k+n)|\lambda| t_{0}}\left\|\widetilde{\varphi}_{k}^{\lambda}\right\|_{L^{\infty}} .
\end{aligned}
$$

Take $t_{0}=\frac{t}{2}$, then

$$
\left.\left.\left|e^{-(2 k+n)|\lambda| t} W_{j} \sqrt{-L} \varphi_{k}^{\lambda}(r) e^{-i \lambda z}\right| \lambda\right|^{n}\left|\leq C t^{-1 / 2}\right| \lambda\right|^{n} \sqrt{(2 k+n)|\lambda|} e^{-(2 k+n)|\lambda| t / 2} .
$$


This implies that the right hand side of (2.18) converges if $\left[W_{j}, \sqrt{-L}\right]$ is replaced by $W_{j} \sqrt{-L}$. Hence we have

$$
W_{j} \sqrt{-L} h_{t}(r, z)=\int_{-\infty}^{\infty} \sum_{k=0}^{\infty} e^{-(2 k+n)|\lambda| t} W_{j} \sqrt{-L} \varphi_{k}^{\lambda}(r) e^{-i \lambda z}|\lambda|^{n} d \lambda .
$$

In order to show (2.18), it remains to consider $\sqrt{-L} W_{j}$. Using right invariant operators and integration by parts, we have

$$
\begin{aligned}
\left|\sqrt{-L} W_{j} \widetilde{\varphi}_{k}^{\lambda}(\mathbf{x})\right| & =\left|\sqrt{-L} W_{j} H_{t_{0}} \widetilde{\varphi}_{k}^{\lambda}(\mathbf{x})\right|=\left|H_{t_{0}} \sqrt{-\hat{L}} \hat{W}_{j} \widetilde{\varphi}_{k}^{\lambda}(\mathbf{x})\right| \\
& =\left|\int_{\mathbb{H}^{n}} h_{t_{0}}(\mathbf{x}, \mathbf{y}) \sqrt{-\hat{L}} \hat{W}_{j} \widetilde{\varphi}_{k}^{\lambda}(\mathbf{y}) d \mathbf{y}\right| \\
& =\left|\int_{\mathbb{H}^{n}} \hat{W}_{j} \sqrt{-\hat{L}} h_{t_{0}}(\mathbf{x}, \mathbf{y}) \widetilde{\varphi}_{k}^{\lambda}(\mathbf{y}) d \mathbf{y}\right| \\
& \leq \int_{\mathbb{H}^{n}}\left|\hat{W}_{j} \sqrt{-\hat{L}} h_{t_{0}}(\mathbf{x}, \mathbf{y})\right| d \mathbf{y}\left\|\widetilde{\varphi}_{k}^{\lambda}\right\|_{L^{\infty}} .
\end{aligned}
$$

By Lemma 2.6, we see that the integral $\int_{\mathbb{H}^{n}}\left|\hat{W}_{j} \sqrt{-\hat{L}} h_{t_{0}}(\mathbf{x}, \mathbf{y})\right| d \mathbf{y}$ converges. This leads to (2.18) with $\left[W_{j}, \sqrt{-L}\right]$ being replaced by $W_{j} \sqrt{-L}$ and hence (2.18).

Remark 2.10. In the same way, we can also prove that

$$
2 i \mathcal{T}_{j} Z h_{t}(r, z)=\int_{-\infty}^{\infty} \sum_{k=0}^{\infty} e^{-(2 k+n)|\lambda| t} 2 i \mathcal{T}_{j} Z \varphi_{k}^{\lambda}(r) e^{-i \lambda z}|\lambda|^{n} d \lambda .
$$

Proof of Proposition 2.8. We first show that

$$
\left[W_{j}, \sqrt{-L}\right] h_{t}(r, z)=2 i \mathcal{T}_{j} Z h_{t}(r, z)
$$

By Lemma 2.9, it suffices to show that, for any $\lambda \in \mathbb{R}$ and any $k \in \mathbb{N}$,

$$
\left[W_{j}, \sqrt{-L}\right] \widetilde{\varphi}_{k}^{\lambda}=2 i \mathcal{T}_{j} Z \widetilde{\varphi}_{k}^{\lambda} .
$$

Before computation, we recall that $Z$ commutes with $L$ and $W_{j}$. Then $L Z \widetilde{\varphi}_{k}^{\lambda}=$ $Z L \widetilde{\varphi}_{k}^{\lambda}=-(2 k+n)|\lambda|$, i.e., $Z \widetilde{\varphi}_{k}^{\lambda}$ is an eigenfunction of the eigenvalue $-(2 k+n)|\lambda|$ for $L$. Thus we have

$$
\begin{aligned}
2 i \mathcal{T}_{j} Z \widetilde{\varphi}_{k}^{\lambda} & =2 i \int_{0}^{+\infty} y P_{y}\left(W_{j} \sqrt{-L}+\sqrt{-L} W_{j}\right) P_{y} Z \widetilde{\varphi}_{k}^{\lambda} d y \\
& =2 i \int_{0}^{+\infty} y e^{-y \sqrt{-L}}\left(W_{j} \sqrt{(2 k+n)|\lambda|}+\sqrt{-L} W_{j}\right) e^{-y \sqrt{(2 k+n)|\lambda|}} Z \widetilde{\varphi}_{k}^{\lambda} d y \\
& =2 i \int_{0}^{+\infty} y e^{-y(\sqrt{-L}+\sqrt{(2 k+n)|\lambda|})}(\sqrt{(2 k+n)|\lambda|}+\sqrt{-L}) W_{j} Z \widetilde{\varphi}_{k}^{\lambda} d y \\
& =\int_{0}^{+\infty} y e^{-y(\sqrt{-L}+\sqrt{(2 k+n)|\lambda|})}(\sqrt{(2 k+n)|\lambda|}+\sqrt{-L})\left(L W_{j}-W_{j} L\right) \widetilde{\varphi}_{k}^{\lambda} d y \\
& =\int_{0}^{+\infty} y e^{-y(\sqrt{-L}+\sqrt{(2 k+n)|\lambda|})}(\sqrt{(2 k+n)|\lambda|}+\sqrt{-L})(L+(2 k+n)|\lambda|) W_{j} \widetilde{\varphi}_{k}^{\lambda} d y \\
& =(\sqrt{(2 k+n)|\lambda|}-\sqrt{-L}) W_{j} \widetilde{\varphi}_{k}^{\lambda}=\left[W_{j}, \sqrt{-L}\right] \widetilde{\varphi}_{k}^{\lambda} .
\end{aligned}
$$

Here, in the third equality, we use (2.12) and the fact that $Z$ commutes with $W_{j}$. 
With this preparation, we can now prove (2.16). By the left invariance, it is enough to prove the equality at $\mathbf{x}=0$. We notice that

$$
\left[W_{j}, \sqrt{-L}\right] f(0)=\lim _{t \rightarrow 0} H_{t}\left[W_{j}, \sqrt{-L}\right] f(0) .
$$

Using integration by parts and also (2.20), we have

$$
\begin{aligned}
H_{t}\left[W_{j}, \sqrt{-L}\right] f(0) & =\int_{\mathbb{H}^{n}} h_{t}(\mathbf{y})\left[W_{j}, \sqrt{-L}\right] f(\mathbf{y}) d \mathbf{y} \\
& =-\int_{\mathbb{H}^{n}}\left[W_{j}, \sqrt{-L}\right] h_{t}(\mathbf{y}) f(\mathbf{y}) d \mathbf{y} \\
& =-\int_{\mathbb{H}^{n}} 2 i \mathcal{T}_{j} Z h_{t}(\mathbf{y}) f(\mathbf{y}) d \mathbf{y} \\
& =\int_{\mathbb{H}^{n}} h_{t}(\mathbf{y}) 2 i \mathcal{T}_{j} Z f(\mathbf{y}) d \mathbf{y}=H_{t} 2 i \mathcal{T}_{j} Z f(0),
\end{aligned}
$$

where the second-to-last equality holds since $Z$ commutes with $L$. This gives us

$$
\lim _{t \rightarrow 0} H_{t}\left[W_{j}, \sqrt{-L}\right] f(0)=2 i \mathcal{T}_{j} Z f(0),
$$

which leads to (2.21) and hence (2.16).

Finally, we conclude that

Proposition 2.11. Let $1 \leq j \leq n$ and $f \in \mathcal{S}\left(\mathbb{H}^{n}\right)$. Then we have

$$
\left\|\left[W_{j}, \sqrt{-L}\right] f\right\|_{p} \leq \sqrt{2}\left(p^{*}-1\right)\|Z f\|_{p} .
$$

Proof. By Proposition 2.8, we have

$$
\left\|\left[W_{j}, \sqrt{-L}\right] f\right\|_{p} \leq 2\left\|\mathcal{T}_{j} Z f\right\|_{p}
$$

It suffices to work on $\mathcal{T}_{j}$. Following the proof of Theorem 1.1, we have GundyVaropoulos type probabilistic representation of $\mathcal{T}_{j}$ as follows

$$
\mathcal{T}_{j} f(x)=-\frac{1}{2} \lim _{y_{0} \rightarrow \infty} \mathbb{E}_{y_{0}}\left(\int_{0}^{\tau} \mathcal{A}_{j}(\nabla, Z)^{\mathrm{T}} Q f\left(\mathcal{Y}_{s}, B_{s}\right)\left(d \beta_{s}, d B_{s}\right) \mid \mathcal{Y}_{\tau}=x\right),
$$

where $\nabla=\left(X_{1}, \cdots, X_{n}, Y_{1}, \cdots, Y_{n}\right),\left(\mathcal{Y}_{t}\right)_{t \geq 0}$ is the diffusion process on $\mathbb{H}^{n}$ with generator $L, \beta_{s}$ is the Brownian motion on $\mathbb{R}^{2 n}$, and $\mathcal{A}_{j}$ is a $(2 n+1) \times(2 n+1)$ matrix as follows:

$a_{j(2 n+1)}=1, a_{(n+j)(2 n+1)}=i, a_{(2 n+1) j}=-1, a_{(2 n+1)(n+j)}=-i ;$ and otherwise 0.

Notice that $\left\|\mathcal{A}_{j}\right\|=\sqrt{2}$, therefore by Theorem 2.5,

$$
\left\|\left[W_{j}, \sqrt{-L}\right] f\right\|_{p} \leq 2\left\|\mathcal{T}_{j} Z f\right\|_{p} \leq 2\left\|\mathcal{A}_{j}\right\| \frac{\left(p^{*}-1\right)}{2}\|Z f\|_{p}=\sqrt{2}\left(p^{*}-1\right)\|Z f\|_{p} .
$$


2.4. Example 3. $\mathbb{S U}(2)$. Consider the Lie group $\mathbb{S U}(2)$, i.e., the group of $2 \times 2$ complex unitary matrices of determinant 1 . Its Lie algebra $\mathfrak{s u}(2)$ consists of $2 \times 2$ complex skew adjoint matrices of trace 0 . A basis of $\mathfrak{s u}(2)$ is formed by the Pauli matrices

$$
X=\left(\begin{array}{cc}
0 & 1 \\
-1 & 0
\end{array}\right), \quad Y=\left(\begin{array}{cc}
0 & i \\
i & 0
\end{array}\right), \quad Z=\left(\begin{array}{cc}
i & 0 \\
0 & -i
\end{array}\right)
$$

for which the commutation relations hold

$$
[X, Y]=2 Z, \quad[Y, Z]=2 X, \quad[Z, X]=2 Y .
$$

Denote by $X, Y, Z$ the left invariant vector fields on $\mathbb{S U}(2)$ corresponding to the Pauli matrices. We shall be interested in the operator

$$
L=X^{2}+Y^{2} .
$$

Let $\left(H_{t}\right)_{t>0}=\left(e^{t L}\right)_{t>0}$ be the heat semigroup generated by $L$ and $h_{t}(\mathbf{x})$ be the corresponding heat kernel at 0 . Let $\left(P_{t}\right)_{t>0}=\left(e^{-t \sqrt{-L}}\right)_{t>0}$ be the Poisson semigroup and $p_{t}$ be the Poisson kernel at 0 . We use the cylindric coordinates introduced in $[22]$

$$
(r, \theta, z) \rightarrow \exp (r \cos \theta X+r \sin \theta Y) \exp (z Z)=\left(\begin{array}{cc}
\cos (r) e^{i z} & \sin (r) e^{i(\theta-z)} \\
-\sin (r) e^{-i(\theta-z)} & \cos (r) e^{i z}
\end{array}\right)
$$

with

$$
0 \leq r \leq \frac{\pi}{2}, \quad \theta \in[0,2 \pi], \quad z \in[-\pi, \pi] .
$$

The heat kernel at 0 depends only on $r$ and $z$, which we denote by $h_{t}(r, z)$. The spectral decomposition of $h_{t}(r, z)$ can be found in [10]: for $t>0,0 \leq r<\frac{\pi}{2}$, $z \in[-\pi, \pi]$

$$
h_{t}(r, z)=\sum_{n=-\infty}^{+\infty} \sum_{k=0}^{+\infty}(2 k+|n|+1) e^{-(4 k(k+|n|+1)+2|n|) t} e^{i n z}(\cos r)^{|n|} P_{k}^{0,|n|}(\cos 2 r) .
$$

Let $W=X+i Y$ be the complex gradient. Then the Lie algebra structure gives us

$$
W L=(L-4 i Z+4) W .
$$

Consider the operator

$$
\mathcal{T}=\int_{0}^{+\infty} y P_{y}(W \sqrt{-L}+\sqrt{-L} W) P_{y} d y .
$$

Proposition 2.12. For any smooth function $f$, there holds

$$
[W, \sqrt{-L}] f=\mathcal{T}(4 i Z+4) f
$$

Proof. Denote $\Phi_{n, k}(r, z)=e^{i n z}(\cos r)^{|n|} P_{k}^{0,|n|}(\cos 2 r)$. Then $\Phi_{n, k}$ is a eigenfunction of $L$ corresponding to the eigenvalue $-\lambda_{n, k}=-4 k(k+|n|+1)-2|n|$. 
Similarly as on Heisenberg groups (details are neglected), it suffices to check (2.23) acting on eigenfunctions $\Phi_{n, k}(r, z)$. We first compute $\mathcal{T} \Phi_{n, k}(r, z)$,

$$
\begin{aligned}
\mathcal{T} \Phi_{n, k} & =\int_{0}^{+\infty} y P_{y}(W \sqrt{-L}+\sqrt{-L} W) P_{y} \Phi_{n, k} d y \\
& =\int_{0}^{+\infty} y e^{-y \sqrt{-L}}\left(W \sqrt{\lambda_{n, k}}+\sqrt{-L} W\right) e^{-y \sqrt{\lambda_{n, k}}} \Phi_{n, k} d y \\
& =\int_{0}^{+\infty} y e^{-y\left(\sqrt{-L}+\sqrt{\lambda_{n, k}}\right)}\left(\sqrt{\lambda_{n, k}}+\sqrt{-L}\right) W \Phi_{n, k} d y \\
& =\left(\sqrt{-L}+\sqrt{\lambda_{n, k}}\right)^{-1} W \Phi_{n, k} .
\end{aligned}
$$

Observe that $[L, Z]=0$, then $L Z \Phi_{n, k}=Z L \Phi_{n, k}=-\lambda_{n, k} Z \Phi_{n, k}$, i.e., $Z \Phi_{n, k}$ is also an eigenfunction of eigenvalue $-\lambda_{n, k}$. Hence the above computation also works for $Z \Phi_{n, k}$ and we obtain that

$$
\mathcal{T}(4 i Z+4) \Phi_{n, k}=\left(\sqrt{-L}+\sqrt{\lambda_{n, k}}\right)^{-1} W(4 i Z+4) \Phi_{n, k} .
$$

Observe also $[W, Z]=2 i W$, which leads to $(4 i Z-4) W=W(4 i Z+4)$. Consequently

$$
\mathcal{T}(4 i Z+4) \Phi_{n, k}=\left(\sqrt{-L}+\sqrt{\lambda_{n, k}}\right)^{-1}(4 i Z-4) W \Phi_{n, k} .
$$

Next compute the commutator acting on $\Phi_{n, k}$, we have

$$
[W, \sqrt{-L}] \Phi_{n, k}=W \sqrt{\lambda_{n, k}} \Phi_{n, k}-\sqrt{-L} W \Phi_{n, k}=\left(\sqrt{\lambda_{n, k}}-\sqrt{-L}\right) W \Phi_{n, k} .
$$

In addition,

$$
\begin{aligned}
\left(\sqrt{-L}+\sqrt{\lambda_{n, k}}\right)\left(\sqrt{\lambda_{n, k}}-\sqrt{-L}\right) W \Phi_{n, k} & =\left(\lambda_{n, k}+L\right) W \Phi_{n, k} \\
& =L W \Phi_{n, k}-W L \Phi_{n, k} \\
& =(4 i Z-4) W \Phi_{n, k},
\end{aligned}
$$

where the last equality is due to (2.22).

Summarize the above three equalities we conclude the proof for (2.23).

As a conclusion, we have

Proposition 2.13. Let $1<p<\infty$. Then for any smooth function $f$,

$$
\|[W, \sqrt{-L}] f\|_{p} \leq 2 \sqrt{2}\left(p^{*}-1\right)\|(i Z+1) f\|_{p} .
$$

Proof. By Proposition 2.12, we have

$$
\|[W, \sqrt{-L}] f\|_{p} \leq\|\mathcal{T}(4 i Z+4) f\|_{p}
$$

Following the proof of Theorem 1.1, we have Gundy-Varopoulos type probabilistic representation of $\mathcal{T}$ as follows

$$
\mathcal{T}_{j} f(x)=-\frac{1}{2} \lim _{y_{0} \rightarrow \infty} \mathbb{E}_{y_{0}}\left(\int_{0}^{\tau} \mathcal{A}_{j}(\nabla, Z)^{\mathrm{T}} Q f\left(\mathcal{Y}_{s}, B_{s}\right)\left(d \beta_{s}, d B_{s}\right) \mid \mathcal{Y}_{\tau}=x\right),
$$

where $\nabla=(X, Y),\left(\mathcal{Y}_{t}\right)_{t \geq 0}$ is the diffusion process on $\mathbb{S U}(2)$ with generator $L, \beta_{s}$ is the Brownian motion on $\mathbb{R}^{2}$, and $\mathcal{A}$ is a $3 \times 3$ matrix as follows:

$$
a_{13}=1, a_{23}=i, a_{31}=-1, a_{32}=-i \text {; and otherwise } 0 .
$$


Notice that $\|\mathcal{A}\|=\sqrt{2}$, therefore by Theorem 2.5,

$$
\begin{aligned}
\|[W, \sqrt{-L}] f\|_{p} & \leq\|\mathcal{T}(4 i Z+4) f\|_{p} \\
& \leq\|\mathcal{A}\| \frac{\left(p^{*}-1\right)}{2}\|(4 i Z+4) f\|_{p} \\
& =2 \sqrt{2}\left(p^{*}-1\right)\|(i Z+1) f\|_{p} .
\end{aligned}
$$

\section{RiESZ TRANSFORMS ON VECTOR BUNDLES}

Our general results are easily generalized in the framework of vector bundles. This framework is more adapted to the study of Riesz transforms vectors.

3.1. A general theorem. Let $\mathbb{M}$ be a $d$-dimensional smooth complete Riemannian manifold and let $\mathcal{E}$ be a finite-dimensional vector bundle over $\mathbb{M}$. We denote by $\Gamma(\mathbb{M}, \mathcal{E})$ the space of smooth sections of this bundle. Let now $\nabla$ denote a metric connection on $\mathcal{E}$. We consider an operator on $\Gamma(\mathbb{M}, \mathcal{E})$ that can be written as

$$
\mathcal{L}=\mathcal{F}+\nabla_{0}+\sum_{i=1}^{d} \nabla_{i}^{2}
$$

where

$$
\nabla_{i}=\nabla_{X_{i}}, \quad 0 \leq i \leq d,
$$

and the $X_{i}$ 's are smooth vector fields on $\mathbb{M}$ and $\mathcal{F}$ is a smooth symmetric and non positive potential (that is a smooth section of the bundle $\operatorname{End}(\mathcal{E})$ ). We will assume that $\mathcal{L}$ is non-positive and essentially self-adjoint on the space $\Gamma_{0}(\mathbb{M}, \mathcal{E})$ of smooth and compactly supported sections. We consider then a first order differential operator $d_{a}$ on $\Gamma(\mathbb{M}, \mathcal{E})$ that can be written as

$$
d_{a}=\sum_{i=1}^{d} a_{i} \nabla_{X_{i}}
$$

where $a_{1}, \cdots, a_{d}$ are smooth sections of the bundle $\operatorname{End}(\mathcal{E})$. Our main assumptions are that

$$
d_{a} \mathcal{L} \eta=\mathcal{L} d_{a} \eta, \quad \eta \in \Gamma(\mathbb{M}, \mathcal{E}),
$$

and that

$$
\left\|d_{a} \eta\right\|^{2} \leq C \sum_{i=1}^{d}\left\|\nabla_{X_{i}} \eta\right\|^{2}, \quad \eta \in \Gamma(\mathbb{M}, \mathcal{E}),
$$

for some constant $C \geq 0$. Several instances of such situations will be illustrated in the sequel. Our main theorem is the following:

Theorem 3.1. For $1<p<\infty$,

$$
\left\|d_{a}(-\mathcal{L})^{-1 / 2} \eta\right\|_{p} \leq 6 C\left(p^{*}-1\right)\|\eta\|_{p} .
$$

The proof follows the same lines as in the previous section. Let $\left(Y_{t}\right)_{t \geq 0}$ be the diffusion process on $\mathbb{M}$ with generator $\sum_{i=1}^{d} X_{i}^{2}+X_{0}$ started from the distribution $\mu$. We assume that $\left(Y_{t}\right)_{t \geq 0}$ is non explosive, thus as before it can then be constructed via the Stratonovitch stochastic differential equation

$$
d Y_{t}=X_{0}(t) d t+\sum_{i=1}^{d} X_{i}\left(Y_{t}\right) \circ d \beta_{t}^{i}
$$


where $\beta_{t}=\left(\beta_{t}^{1}, \cdots, \beta_{t}^{d}\right)$ is the Brownian motion on $\mathbb{R}^{d}$ with generator $\sum_{i=1}^{d} \frac{\partial^{2}}{\partial x_{i}^{2}}$.

Let $\left(B_{t}\right)_{t \geq 0}$ be a one-dimensional Brownian motion on $\mathbb{R}$, with generator $\frac{d^{2}}{d y^{2}}$ starting from $y_{0}>0$, which is independent from $\left(Y_{t}\right)_{t \geq 0}$. Then $\mathbb{E}\left(B_{t}^{2}\right)=2 t$. Set the stopping time

$$
\tau=\inf \left\{t>0, B_{t}=0\right\} .
$$

We denote by $H_{t}=e^{t L}$ the heat semigroup and by $P_{y}=e^{-y \sqrt{-L}}$ the Poisson semigroup. In that framework, there is a well known Feynman-Kac representation for the semigroup $H_{t}$.

More precisely, consider the stochastic parallel transport along $Y_{t}, \theta_{t}: \mathcal{E}_{Y_{t}} \rightarrow \mathcal{E}_{Y_{0}}$ and the multiplicative functional $\left(\mathcal{M}_{t}\right)_{t \geq 0}$, solution of the equation

$$
\frac{d \mathcal{M}_{t}}{d t}=\mathcal{M}_{t} \theta_{t} \mathcal{F} \theta_{t}^{-1}, \quad \mathcal{M}_{0}=\mathbf{I d} .
$$

By the Feynman-Kac formula, the heat semigroup $H_{t}$ acting on $\eta \in \Gamma_{0}^{\infty}(\mathbb{M}, \mathcal{E})$ can then be written as

$$
H_{t} \eta(x)=\mathbb{E}_{x}\left(\mathcal{M}_{t} \theta_{t} \eta\left(Y_{t}\right)\right) .
$$

For $\eta \in \Gamma_{0}^{\infty}(\mathbb{M}, \mathcal{E})$, denote

$$
Q \eta(x, y)=P_{y} \eta(x)=e^{-y \sqrt{-\mathcal{L}}} \eta(x),
$$

and

$$
M_{s}^{\eta}=\mathcal{M}_{s \wedge \tau} \theta_{s \wedge \tau} Q \eta\left(Y_{s \wedge \tau}, B_{s \wedge \tau}\right) .
$$

As in Lemma 2.2, we can easily prove that $M^{\eta}$ is a martingale. We have then the following Gundy-Varopoulos type representation :

Lemma 3.2. Let $\eta \in \Gamma_{0}^{\infty}(\mathbb{M}, \mathcal{E})$. For almost all $x \in \mathbb{M}$, we have

$d_{a}(-\mathcal{L})^{-1 / 2} \eta(x)=-2 \lim _{y_{0} \rightarrow \infty} \mathbb{E}_{y_{0}}\left(\theta_{\tau}^{-1} \mathcal{M}_{\tau}^{*} \int_{0}^{\tau}\left(\mathcal{M}_{s}^{*}\right)^{-1} \theta_{s} d_{a} Q f\left(Y_{s}, B_{s}\right) d B_{s} \mid Y_{\tau}=x\right)$.

Proof. Let $\alpha \in \Gamma_{0}^{\infty}(\mathbb{M}, \mathcal{E})$ and $\alpha \in \Gamma_{0}^{\infty}(\mathbb{M}, \mathcal{E})$. Note that

$$
M_{\tau}^{\eta}=\mathcal{M}_{\tau} \theta_{\tau} \alpha\left(Y_{\tau}\right)
$$

By Itô isometry, we have

$$
\begin{aligned}
& \int_{\mathbb{M}}\left\langle\alpha(x), \mathbb{E}\left(\theta_{\tau}^{-1} \mathcal{M}_{\tau}^{*} \int_{0}^{\tau}\left(\mathcal{M}_{s}^{*}\right)^{-1} \theta_{s} d_{a} Q \eta\left(Y_{s}, B_{s}\right) d B_{s} \mid Y_{\tau}=x\right)\right\rangle d \mu(x) \\
& =\mathbb{E}_{y_{0}}\left(\left\langle\mathcal{M}_{\tau} \theta_{\tau} \alpha\left(Y_{\tau}\right), \int_{0}^{\tau}\left(\mathcal{M}_{s}^{*}\right)^{-1} \theta_{s} d_{a} Q \eta\left(Y_{s}, B_{s}\right) d B_{s}\right\rangle\right) \\
& =2 \mathbb{E}_{y_{0}}\left(\int_{0}^{\tau}\left\langle\partial_{y} Q \alpha\left(Y_{s}, B_{s}\right), d_{a} Q \eta\left(Y_{s}, B_{s}\right)\right\rangle d s\right) \\
& =2 \int_{\mathbb{M}} \int_{0}^{\infty}\left(y_{0} \wedge y\right)\left\langle\partial_{y} Q \alpha(x, y), d_{a} Q \eta(x, y)\right\rangle d y d \mu(x) .
\end{aligned}
$$

The last equality follows from the facts that the Green function of the Brownian motion is killed at 0 and $Y_{s}$ is distributed according to $\mu$. Using finally the commutation between $d_{a}$ and $\mathcal{L}$ one deduces

$$
2 \int_{\mathbb{M}} \int_{0}^{\infty} y\left\langle\partial_{y} Q \alpha(x, y), d_{a} Q \eta(x, y)\right\rangle d y d \mu(x)=-\frac{1}{2} \int_{\mathbb{M}}\left\langle\alpha(x), d_{a}(-\mathcal{L})^{-1 / 2} \eta(x)\right\rangle d \mu(x) .
$$

The proof of Theorem 3.1 now follows the lines of the proof of Theorem 1.1. 
3.2. Example 1. The Riesz transform on forms. Let $\mathbb{M}$ be a $d$-dimensional smooth, oriented, complete and stochastically complete Riemannian manifold. We first briefly recall some basic facts on Fermion calculus on the exterior algebra of a finite dimensional vector space, as can be found in Section 2.2.2 of [40]. Let $V$ be a $d$-dimensional Euclidean vector space. We denote $V^{*}$ its dual and

$$
\wedge V^{*}=\bigoplus_{k \geq 0} \wedge^{k} V^{*}
$$

the exterior algebra. If $u \in V^{*}$, we denote $a_{u}^{*}$ the map $\wedge V^{*} \rightarrow \wedge V^{*}$, such that $a_{u}^{*}(\omega)=u \wedge \omega$. The dual map is denoted $a_{u}$. Let now $\theta_{1}, \ldots, \theta_{d}$ be an orthonormal basis of $V^{*}$. We denote $a_{i}=a_{\theta_{i}}$. We have the basic rules of Fermion calculus

$$
\left\{a_{i}, a_{j}\right\}=0,\left\{a_{i}^{*}, a_{j}^{*}\right\}=0,\left\{a_{i}, a_{j}^{*}\right\}=\delta_{i j},
$$

where $\{\cdot, \cdot\}$ stands for the anti-commutator: $\left\{a_{i}, a_{j}\right\}=a_{i} a_{j}+a_{j} a_{i}$. If $I$ and $J$ are two words with $1 \leq i_{1}<\cdots<i_{k} \leq d$ and $1 \leq j_{1}<\cdots<j_{l} \leq d$, we denote

$$
A_{I J}=a_{i_{1}}^{*} \cdots a_{i_{k}}^{*} a_{j_{1}} \cdots a_{j_{l}} \text {. }
$$

The family of all the possible $A_{I J}$ forms a basis of the $2^{2 d}$-dimensional vector space End $\left(\wedge V^{*}\right)$. We can carry the Fermionic construction on the tangent spaces of the manifold $\mathbb{M}$. Let $e_{i}$ be a local orthonormal frame and let $\theta_{i}$ be its dual frame. In that frame, we can express the exterior derivative as

$$
d=\sum_{i} a_{i}^{*} \nabla_{e_{i}}
$$

Let us therefore observe that $\|d \eta\|^{2} \leq \sum_{i=1}^{d}\left\|\nabla_{e_{i}} \eta\right\|^{2}$. The curvature endomorphism (Weitzenböck curvature) is then defined by

$$
\mathcal{F}=-\sum_{i j k l} R_{i j k l} a_{i}^{*} a_{j} a_{k}^{*} a_{l}
$$

where

$$
R_{i j k l}=\left\langle R\left(e_{j}, e_{k}\right) e_{l}, e_{i}\right\rangle
$$

with $R$ Riemannian curvature of $\mathbb{M}$. The celebrated Weitzenböck formula writes

$$
\mathcal{L}=\Delta-\mathcal{F},
$$

where $\mathcal{L}=-d d^{*}-d^{*} d$ is the Hodge-DeRham Laplacian and $\Delta$ the Bochner Laplacian. Let us recall that if $e_{i}$ is a local orthonormal frame, we have the following explicit formula for $\Delta$ :

$$
\Delta=\sum_{i=1}^{d}\left(\nabla_{e_{i}} \nabla_{e_{i}}-\nabla_{\nabla_{e_{i}} e_{i}}\right),
$$

where $\nabla$ is the Levi-Civita connection. The following theorem is then an application of Theorem 3.1.

Theorem 3.3. Assume $\mathcal{F} \geq 0$, then

$$
\left\|d(-\mathcal{L})^{-1 / 2} \eta\right\|_{p} \leq 6\left(p^{*}-1\right)\|\eta\|_{p}
$$

Remark 3.4. Let us observe that the expressions 3.25 and 3.26 are only defined locally in a given frame, however Lemma 3.2 is coordinate free and therefore holds in the present setting (see Theorem 3.2 in [30]). 
3.3. Example 2. The Riesz transform on spinors. We first review some basic constructions in spin geometry. Let $V$ be an oriented $d$ dimensional Euclidean space. We assume that the dimension $d$ is even. The Clifford algebra $\mathbf{C l}(V)$ over $V$ is the algebra

$$
\mathbf{T}(V)=\mathbb{R} \oplus V \oplus(V \otimes V) \oplus \cdots
$$

quotient by the relations

$$
u \otimes v+v \otimes u+2\langle u, v\rangle 1=0 .
$$

Let $e_{1}, \ldots, e_{d}$ be an oriented basis of $V$. The family

$$
e_{i_{1}} \ldots e_{i_{k}}, \quad 0 \leq k \leq d, \quad 1 \leq i_{1}<\ldots<i_{k} \leq d,
$$

forms a basis of the vector space $\mathbf{C l}(V)$ which is therefore of dimension $2^{d}$. In $\mathbf{T}(V)$ we can distinguish elements that are even from elements that are odd. This leads to a decomposition:

$$
\mathbf{C l}(V)=\mathbf{C l}^{-}(V) \oplus \mathbf{C l}^{+}(V),
$$

with $V \subset \mathbf{C l}^{-}(V)$. A Clifford module is a vector space $E$ over $\mathbb{R}$ (or $\mathbb{C}$ ) that is also a $\mathbf{C l}(V)$-module and that admits a direct sum decomposition

$$
E=E^{-} \oplus E^{+}
$$

with

$$
\mathrm{Cl}^{-}(V) \cdot E^{-} \subset E^{-}, \quad \mathbf{C l}^{+}(V) \cdot E^{+} \subset E^{+} .
$$

It can be shown that there is a unique Clifford module $S$, called the spinor module over $V$ such that:

$$
\operatorname{End}(S) \simeq \mathbb{C} \otimes \mathbf{C l}(V) .
$$

In particular $\operatorname{dim} S=2^{\frac{d}{2}}$. If $\psi \in \mathfrak{s o}(V)$, that is if $\psi: V \rightarrow V$ is a skew-symmetric map, we define

$$
D \psi=\frac{1}{2} \sum_{1 \leq i<j \leq d}\left\langle\psi\left(e_{i}\right), e_{j}\right\rangle e_{i} e_{j} \in \mathbf{C l}(V),
$$

and observe that $D\left[\psi_{1}, \psi_{2}\right]=\left[D \psi_{1}, D \psi_{2}\right]$. The set $\mathbf{C l}^{2}(V)=D \mathfrak{s o}(V)$ is therefore a Lie algebra. The Lie group $\operatorname{Spin}(V)$ is the group obtained by exponentiating $\mathbf{C l}^{2}(V)$ inside the Clifford algebra $\mathbf{C l}(V)$; It is the two-fold universal covering of the orthogonal group $\mathbf{S O}(V)$. It can also be described as the set of $a \in \mathbf{C l}(V)$ such that:

$$
a=v_{1} \ldots v_{2 k}, \quad 1 \leq k \leq \frac{d}{2}, \quad v_{i} \in V, \quad\left\|v_{i}\right\|=1 .
$$

We now come back to the manifold setting and carry the above constructions on the cotangent spaces of a spin manifold. So, let $\mathbb{M}$ be a $d$-dimensional, oriented, complete and stochastically complete Riemannian manifold. We assume that $d$ is even. We furthermore assume that $\mathbb{M}$ admits a spin structure: That is, there exists a principal bundle on $\mathbb{M}$ with structure group $\operatorname{Spin}\left(\mathbb{R}^{d}\right)$ such that the bundle charts are compatible with the universal covering $\operatorname{Spin}\left(\mathbb{R}^{d}\right) \rightarrow \mathbf{S O}\left(\mathbb{R}^{d}\right)$. This bundle will be denoted $\mathcal{S P}(\mathbb{M})$ and $\pi$ will denote the canonical surjection. The spin bundle $\mathcal{S}$ over $\mathbb{M}$ is the vector bundle such that for every $x \in \mathbb{M}, \mathcal{S}_{x}$ is the spinor module over the cotangent space $\mathbf{T}_{x}^{*} \mathbb{M}$. At each point $x$, there is therefore a natural action of $\mathbf{C l}\left(\mathbf{T}_{x}^{*} \mathbb{M}\right) \simeq \operatorname{End}\left(\mathcal{S}_{x}\right)$; this action will be denoted by $\mathbf{c}$. On $\mathcal{S}$, there is a canonical 
elliptic first-order differential operator called the Dirac operator and denoted D. In a local orthonormal frame $e_{i}$, with dual frame $e_{i}^{*}$, the Dirac operator is given by

$$
\mathbf{D}=\sum_{i=1}^{d} c\left(e_{i}^{*}\right) \nabla_{e_{i}},
$$

where $\nabla$ is the Levi-Civita connection. As a consequence, $\|\mathbf{D} \eta\|^{2} \leq \sum_{i=1}^{d}\left\|\nabla_{e_{i}} \eta\right\|^{2}$. We also have an analogue of Weitzenböck formula which is the celebrated Lichnerowicz formula (see Theorem 3.52 in [13]):

$$
-\mathbf{D}^{2}=\Delta-\frac{s}{4}
$$

where $s$ is the scalar curvature of $\mathbb{M}$ and $\Delta$ is given in a local orthonormal frame $e_{i}$ by

$$
\Delta=\sum_{i=1}^{d}\left(\nabla_{e_{i}} \nabla_{e_{i}}-\nabla_{\nabla_{e_{i}} e_{i}}\right) .
$$

The following theorem is then an application of Theorem 3.1.

Theorem 3.5. Assume that the scalar curvature $s \geq 0$, then

$$
\left\|\mathbf{D}\left(-\mathbf{D}^{2}\right)^{-1 / 2} \eta\right\|_{p} \leq 6\left(p^{*}-1\right)\|\eta\|_{p} .
$$

\section{REFERENCES}

[1] G. Alexopoulos. An application of homogenization theory to harmonic analysis: Harnack inequalities and Riesz transforms on Lie groups of polynomial growth. Canad. J. Math., 44(4):691-727, 1992. 2

[2] N. Arcozzi. Riesz transforms on compact Lie groups, spheres and Gauss space. Ark. Mat., 36(2):201-231, 1998. 3, 9

[3] P. Auscher, T. Coulhon, X. T. Duong, and S. Hofmann. Riesz transform on manifolds and heat kernel regularity. Ann. Sci. École Norm. Sup. (4), 37(6):911-957, 2004. 2

[4] P. Auscher, A. McIntosh, and E. Russ. Hardy spaces of differential forms on Riemannian manifolds. J. Geom. Anal., 18(1):192-248, 2008. 2

[5] R. Bañuelos and F. Baudoin. Martingale transforms and their projection operators on manifolds. Potential Anal., 38(4):1071-1089, 2013. 9

[6] R. Bañuelos and A. Osȩkowski. Sharp martingale inequalities and applications to Riesz transforms on manifolds, Lie groups and Gauss space. J. Funct. Anal., 269(6):1652-1713, 2015. 7

[7] R. Bañuelos and G. Wang. Sharp inequalities for martingales with applications to the Beurling-Ahlfors and Riesz transforms. Duke Math. J., 80(3):575-600, 1995. 1, 3, 7

[8] D. Bakry. Étude des transformations de Riesz dans les variétés riemanniennes à courbure de Ricci minorée. In Séminaire de Probabilités, XXI, volume 1247 of Lecture Notes in Math., pages 137-172. Springer, Berlin, 1987. 2

[9] D. Bakry, F. Baudoin, M. Bonnefont, and D. Chafaï. On gradient bounds for the heat kernel on the Heisenberg group. J. Funct. Anal., 255(8):1905-1938, 2008. 10, 11

[10] F. Baudoin and M. Bonnefont. The subelliptic heat kernel on SU(2): representations, asymptotics and gradient bounds. Math. Z., 263(3):647-672, 2009. 15

[11] F. Baudoin and M. Bonnefont. Reverse Poincaré inequalities, isoperimetry, and Riesz transforms in Carnot groups. Nonlinear Anal., 131:48-59, 2016. 2

[12] F. Baudoin and N. Garofalo. A note on the boundedness of Riesz transform for some subelliptic operators. Int. Math. Res. Not. IMRN, (2):398-421, 2013. 2

[13] N. Berline, E. Getzler, and M. Vergne. Heat kernels and Dirac operators, volume 298 of Grundlehren der Mathematischen Wissenschaften [Fundamental Principles of Mathematical Sciences]. Springer-Verlag, Berlin, 1992. 21

[14] A. Carbonaro and O. Dragičević. Bellman function and linear dimension-free estimates in a theorem of Bakry. J. Funct. Anal., 265(7):1085-1104, 2013. 2 
[15] G. Carron. Riesz transforms on connected sums. Ann. Inst. Fourier (Grenoble), 57(7):23292343, 2007. Festival Yves Colin de Verdière. 2

[16] G. Carron, T. Coulhon, and A. Hassell. Riesz transform and $L^{p}$-cohomology for manifolds with Euclidean ends. Duke Math. J., 133(1):59-93, 2006. 2

[17] L. Chen, T. Coulhon, J. Feneuil, and E. Russ. Riesz transform for $1 \leq p \leq 2$ without Gaussian heat kernel bound. J. Geom. Anal., 27(2):1489-1514, 2017. 2

[18] P. Chen, J. Magniez, and E. M. Ouhabaz. The Hodge-de Rham Laplacian and $L^{p_{-}}$ boundedness of Riesz transforms on non-compact manifolds. Nonlinear Anal., 125:78-98, 2015. 2

[19] T. Coulhon and X. T. Duong. Riesz transforms for $1 \leq p \leq 2$. Trans. Amer. Math. Soc., 351(3):1151-1169, 1999. 2

[20] T. Coulhon and X. T. Duong. Riesz transform and related inequalities on noncompact Riemannian manifolds. Comm. Pure Appl. Math., 56(12):1728-1751, 2003. 2

[21] T. Coulhon, D. Müller, and J. Zienkiewicz. About Riesz transforms on the Heisenberg groups. Math. Ann., 305(2):369-379, 1996. 2

[22] M. Cowling and A. Sikora. A spectral multiplier theorem for a sublaplacian on SU(2). Math. Z., 238(1):1-36, 2001. 15

[23] B. Devyver. A Gaussian estimate for the heat kernel on differential forms and application to the Riesz transform. Math. Ann., 358(1-2):25-68, 2014. 2

[24] C. Guillarmou and D. A. Sher. Low energy resolvent for the Hodge Laplacian: applications to Riesz transform, Sobolev estimates, and analytic torsion. Int. Math. Res. Not. IMRN, (15):6136-6210, 2015. 2

[25] T. Iwaniec and G. Martin. Riesz transforms and related singular integrals. J. Reine Angew. Math., 473:25-57, 1996. 1, 3

[26] E. Lenglart, D. Lépingle, and M. Pratelli. Présentation unifiée de certaines inégalités de la théorie des martingales. In Seminar on Probability, XIV (Paris, 1978/1979) (French), volume 784 of Lecture Notes in Math., pages 26-52. Springer, Berlin, 1980. With an appendix by Lenglart. 8

[27] H.-Q. Li. La transformation de Riesz sur les variétés coniques. J. Funct. Anal., 168(1):145238, 1999. 2

[28] H.-Q. Li. Analyse sur les variétés cuspidales. Math. Ann., 326(4):625-647, 2003. 2

[29] H.-Q. Li and N. Lohoué. Transformées de Riesz sur une classe de variétés à singularités coniques. J. Math. Pures Appl. (9), 82(3):275-312, 2003. 2

[30] X.-D. Li. Martingale transforms and $L^{p}$-norm estimates of Riesz transforms on complete Riemannian manifolds. Probab. Theory Related Fields, 141(1-2):247-281, 2008. 2, 3, 4, 19

[31] X.-D. Li. $L^{p}$-estimates and existence theorems for the $\bar{\partial}$-operator on complete Kähler manifolds. Adv. Math., 224(2):620-647, 2010. 2, 3

[32] X.-D. Li. Riesz transforms on forms and $L^{p}$-Hodge decomposition on complete Riemannian manifolds. Rev. Mat. Iberoam., 26(2):481-528, 2010. 2, 3

[33] X.-D. Li. Erratum to: Martingale transforms and $L^{p}$-norm estimates of Riesz transforms on complete Riemannian manifolds [mr2372971]. Probab. Theory Related Fields, 159(1-2):405408, 2014. 2, 3

[34] X.-D. Li. Erratum to "Riesz transforms on forms and $L^{p}$-Hodge decomposition on complete Riemanian manifolds" [mr2677005]. Rev. Mat. Iberoam., 30(1):369-370, 2014. 2, 3

[35] P. A. Meyer. Démonstration probabiliste de certaines inégalités de Littlewood-Paley. I. Les inégalités classiques. pages 125-141. Lecture Notes in Math., Vol. 511, 1976. 5

[36] P.-A. Meyer. Transformations de Riesz pour les lois gaussiennes. In Seminar on probability, XVIII, volume 1059 of Lecture Notes in Math., pages 179-193. Springer, Berlin, 1984. 2

[37] D. Müller, M. M. Peloso, and F. Ricci. Analysis of the Hodge Laplacian on the Heisenberg group. Mem. Amer. Math. Soc., 233(1095):vi+91, 2015. 2

[38] S. K. Pichorides. On the best values of the constants in the theorems of M. Riesz, Zygmund and Kolmogorov. Studia Math., 44:165-179. (errata insert), 1972. Collection of articles honoring the completion by Antoni Zygmund of 50 years of scientific activity, II. 3

[39] L. Roncal and S. Thangavelu. Hardy's inequality for fractional powers of the sublaplacian on the Heisenberg group. Adv. Math., 302:106-158, 2016. 11

[40] S. Rosenberg. The Laplacian on a Riemannian manifold, volume 31 of London Mathematical Society Student Texts. Cambridge University Press, Cambridge, 1997. An introduction to analysis on manifolds. 19 
[41] E. M. Stein. Topics in harmonic analysis related to the Littlewood-Paley theory. Annals of Mathematics Studies, No. 63. Princeton University Press, Princeton, N.J.; University of Tokyo Press, Tokyo, 1970. 1

[42] R. S. Strichartz. Analysis of the Laplacian on the complete Riemannian manifold. J. Funct. Anal., 52(1):48-79, 1983. 1, 2

[43] J. van Neerven and R. Versendaal. $L^{p}$-analysis of the Hodge-Dirac operator associated with Witten Laplacians on complete Riemannian manifolds. J. Geom. Anal., 2017. 2

Department of Mathematics, Purdue University, West Lafayette, IN 47907, USA

E-mail address: banuelos@math.purdue.edu

Department of Mathematics, University of Connecticut, Storrs, CT 06269

E-mail address: fabrice.baudoin@uconn.edu

Department of Mathematics, University of Connecticut, Storrs, CT 06269

E-mail address: li.4.chen@uconn.edu 